prevents the vaccine from being immunosuppressive itself. In our studies, we demonstrate that VLVs are able to induce strong, broad, and long-lasting ERV Env specific CD8 $+\mathrm{T}$ cell by flow cytometry and antibody responses by ELISA in mice. Furthermore, the modified vaccine is of special interest to future research as it proved to significantly delay mouse tumor growth in a therapeutic setup. Nevertheless, we now need to address the principal host related developmental uncertainties in translating our achievements into the clinical setting. This goal can be accomplished by raising human $\mathrm{T}$ cells capable of targeting human cancers ex vivo. Furthermore, to support the translation of our work, we tested the ability to rise adaptive responses upon vaccination in non-human primates (NHP) which endogenously express ERVs similar to humans (in collaboration with IPB University, Indonesia). Fellowship granted by Innovation Fund Denmark.

Disclosure Information J. Daradoumis: A. Employment (full or part-time); Significant; InProTher Aps. B. Research Grant (principal investigator, collaborator or consultant and pending grants as well as grants already received); Significant; Innovation Fund Denmark. P.J. Holst: A. Employment (full or parttime); Significant; InProTher Aps. E. Ownership Interest (stock, stock options, patent or other intellectual property); Significant; InProTher. K.N. Nielsen: A. Employment (full or part-time); Significant; InProTher Aps.

\section{P04.09 DEVELOPMENT OF A DENDRITIC CELL VACCINE AGAINST HEPATOCELLULAR CARCINOMA USING VSV-NDV}

1) Gold*, ${ }^{1} \mathrm{~L}$ Hanesch, ${ }^{1} \mathrm{~T}$ Krabbe, ${ }^{1} \mathrm{C}$ Lozano-Simon, ${ }^{2} \mathrm{G}$ Hänel, ${ }^{2} \mathrm{M}$ Rothe, ${ }^{3} \mathrm{~A}$ Klopp, ${ }^{4} \mathrm{~S}$ Göttert, ${ }^{4} \mathrm{~S}$ Heidegger, ${ }^{3} \mathrm{U}$ Protzer, ${ }^{2} \mathrm{M}$ Subklewe, ${ }^{1} \mathrm{~J}$ Altomonte. ${ }^{1}$ Department of Internal Medicine II, Klinikum rechts der Isar, Technical University of Munich, Munich, Germany; ${ }^{2}$ Department of Medicine III, University Hospital, LMU Munich, Munich, Germany; ${ }^{3}$ Institute of Virology at the Technical University of Munich, Munich, Germany; ${ }^{4}$ Department of Internal Medicine III, Klinikum rechts der Isar, Technical University of Munich, Munich, Germany

\subsection{6/jitc-2020-ITOC7.77}

Background Activated Dendritic cells (DC) are the immune system's allrounder: they initiate innate and adaptive immune responses; they induce instant immune reactions as well as immunologic memory. Therefore, there is growing interest in using them as a potential anticancer vaccine. ${ }^{1}$ Here we use the beneficial immune-stimulatory properties of the novel oncolytic hybrid virus VSV-NDV to create a DC vaccine against hepatocellular carcinoma. ${ }^{2}$

In our therapeutic approach, a sample of the patient's tumor cells is lysed in vitro with VSV-NDV (=oncolysate). The patient's DCs are then co-cultured in vitro with the oncolysate in order to activate them and load them with tumor antigens. In the end, the stimulated DCs are injected into the patient, where they can lead to a personalized and broad antitumor immune response.

Materials and Methods To investigate the potential of the approach in a cell culture system, human monocyte-derived dendritic cells were generated from PBMCs of healthy donors and incubated with VSV-NDV-lysed HepG2 hepatoma cells. Afterwards their state of activation was investigated via flow cytometry and cytokine measurement, whereas their functionality was assessed in co-culture with T-cells. In a murine system, dendritic cells were generated

from bone marrow stem cells, incubated with a VSV-NDVlysed murine HCC clone and investigated as in the human system.

Results Flow cytometry of oncolysate-stimulated DCs showed a significant upregulation of the activation markers CD86, MHC-I, MHC-II and PD-L1 ( $\mathrm{p}<0.05)$. Moreover, these stimulated DCs released increased amounts of cytokines. Upon co-culture of the DCs with T-cells, an elevated secretion of IFN $\gamma$ by the T-cells, as well as an upregulation of T-cell activation markers could be shown, demonstrating the functional potential of the oncolysate-stimulated DCs. These results apply to both the human and the murine system.

Conclusions Our in vitro data demonstrates that the oncolysate-stimulated human and murine DCs are not only activated, but furthermore have a high functional potential. Further in vitro-experiments will be necessary to translate the process to patient-derived samples, whereas murine in vivo-experiments will give further insights into the effect of the therapeutic approach.

\title{
REFERENCES
}

1. Lichtenegger FS, Rothe M, Schnorfeil FM, Deiser K, Krupka C, Augsberger C, Schluter M, Neitz J, Subklewe M. Targeting LAG-3 and PD-1 to Enhance T Cell Activation by Antigen-Presenting Cells. Front Immunol 2018.

2. Abdullahi $S$, Jäkel $M$, Behrend $S J$, Steiger $K$, Topping $G$, Krabbe $T$, Colombo $A$, Sandig V, Schiergens TS, Thasler WE, Werner J, Lichtenthaler SF, Schmid RM, Ebert 0, Altomonte J. A Novel Chimeric Oncolytic Virus Vector for Improved Safety and Efficacy as a Platform for the Treatment of Hepatocellular Carcinoma. J Virol 2018.

Disclosure Information J. Gold: None. L. Hanesch: None. T. Krabbe: None. C. Lozano-Simon: None. G. Hänel: None. M. Rothe: None. A. Klopp: None. S. Göttert: None. S. Heidegger: None. U. Protzer: None. M. Subklewe: None. J. Altomonte: None.

\section{P05 Precision Medicine Meets Immunotherapy (Immuno-Monitoring)}

\section{P05.01 COMPARATIVE ANALYSIS OF RNA VERSUS DNA AS INPUT MATERIAL FOR IGH REPERTOIRE SEQUENCING PANELS FOR IMMUNO-ONCOLOGY APPLICATIONS AND RARE CLONE DETECTION}

${ }^{1}$ GM Lowman*, ' ${ }^{1}$ P Pickle, ${ }^{1} \mathrm{M}$ Toro, ${ }^{1} \mathrm{~J}$ Chang, ${ }^{1} \mathrm{D}$ Topacio-Hall, ${ }^{2} \mathrm{~T}$ Looney. ${ }^{1}$ Thermo Fisher Scientific, Carlsbad, CA, USA; ${ }^{2}$ Thermo Fisher Scientific, Austin, TX, USA

\subsection{6/jitc-2020-ITOC7.78}

Background Recent progress in tumor immunotherapies have shown the importance of next generation sequencing (NGS) T cell repertoire profiling to characterize $\mathrm{T}$ cell immune response to treatment. Understanding the role of the $\mathrm{B}$ cell repertoire upon stimulation of the immune system by checkpoint blockade is paramount for immunotherapeutic approaches in treatment of B cell malignancies, as well as understanding B cell function within traditional $\mathrm{I} / \mathrm{O}$ strategies. The ability to detect low frequency B cell clones enables numerous hematology/ oncology research applications, including identification of potential biomarkers and minimal residual disease (MRD) research. Historically, efforts to track the frequency of malignant B cells by IGH chain sequencing have utilized DNA input given potential challenges in accurately quantifying 
template copy number from RNA data owing to B cell subtype specific variation in the expression of the $\mathrm{B}$ cell receptor. Hypothetically, however, RNA input based monitoring could be advantageous both owing to reduced input requirements and superior ability to detect $\mathrm{B}$ call malignancies of plasmablast and plasma cell origin, where the BCR is robustly expressed. Here we compared the ability of RNA and DNA based IGH chain sequencing to detect Burkitt's Lymphoma cell lines and Chronic Lymphocytic Leukemia samples at a frequency of $10^{-6}$ from peripheral blood.

Materials and Methods Here we present performance for rare clone detection utilizing the Ion Oncomine ${ }^{\mathrm{TM}}$ BCR IGH-SR assay and the Ion Oncomine ${ }^{\mathrm{TM}}$ BCR IGH-LR assay. These assays use multiplex primers targeting all known IGH germline variable genes in the framework 1 (FR1) or framework 3 (FR3) regions of the $\mathrm{B}$ cell receptor using either DNA or RNA as input. To evaluate detection sensitivity of the IGH-SR assay we utilized DNA or RNA from Burkitt's lymphoma cell lines as well as clinical chronic lymphocytic leukemia (CLL) samples controllably added to a background of peripheral blood leukocytes (PBL) by mass ratio to create specimens with a known target B cell frequency. Automated downsampling analysis was used to confirm libraries were sequenced to saturation. Library preparation and analysis was performed in replicate to quantify sensitivity of detection.

Results For each cell line, we prepared and sequenced (1) 30 libraries derived from amplification of $2 \mathrm{ug}$ gDNA spiked with 2pg cell line gDNA and (2) 10 libraries derived from amplification of $100 \mathrm{ng}$ RNA spiked with $0.1 \mathrm{pg}$ cell line total RNA. The Burkitt's lymphoma cell line and CLL samples were detected in 10/30 and 8/30 libraries respectively, consistent with the performance of orthologous DNA-based sequencing approaches. For RNA libraries, the Burkitt's lymphoma and CLL samples were detected in each library (10/10 and 10/10, respectively).

Conclusions Here we demonstrate the ability to detect B cell clones down to $10^{-6}$ from gDNA and RNA inputs utilizing the Ion Oncomine ${ }^{\mathrm{TM}}$ BCR IGH-SR assay. Feasibility for rare clone detection is shown in gDNA or RNA enabling $\mathrm{B}$ cell minimal residual disease research, and high sensitivity characterization the $\mathrm{B}$ cell role in response to checkpoint blockade within the tumor microenvironment. Importantly, we find that RNA based IGH sequencing may significantly reduce input requirements for rare clone detection, potentially enabling routine detection of clones at $10^{-6}$ frequency from a single library.

Disclosure Information G.M. Lowman: A. Employment (full or part-time); Significant; ThermoFisher Scientific. L. Pickle: A. Employment (full or part-time); Significant; ThermoFisher Scientific. M. Toro: A. Employment (full or part-time); Significant; ThermoFisher Scientific. J. Chang: A. Employment (full or part-time); Significant; ThermoFisher Scientific. D. TopacioHall: A. Employment (full or part-time); Significant; ThermoFisher Scientific. T. Looney: A. Employment (full or parttime); Significant; ThermoFisher Scientific.

\section{P05.02 AN INTEGRATED VIRTUAL TISSUE PLATFORM FOR INCORPORATING EXERCISE ONCOLOGY INTO IMMUNOTHERAPY}

A Hagar*, J Aponte Serrano. Indiana University Bloomington, Bloomington, IN, USA
We introduce a novel in silico platform for simulating solid tumor growth and anti-tumor immune response. We present the model, test the sensitivity and robustness of its parameters, and calibrate it with pre-clinical and clinical data from exercise oncology experiments which offer a natural biological backdrop for modulation of anti-tumor immune response. We then perform two virtual experiments with the model that demonstrate its usefulness in guiding pre-clinical and clinical studies on immunotherapy. The first virtual experiment probes the intricate dynamics in the tumor microenvironment between the tumor and the infiltrating immune cells. Such dynamics is difficult to probe during a pre-clinical study as it requires significant of redundancy in lab animals and is time and labor intensive. The result is a time series of spatiotemporal observational 'windows' into the tumor microenvironment that can serve as a platform to test several mechanistic hypotheses on the role and dynamics of different immune cells in ant-tumor immune response. The second virtual experiment shows how dosage and frequency of immunotherapy drugs can be optimized based on the aerobic fitness of the patient, so that possible adverse side effects of the treatment can be minimized.

Disclosure Information A. Hagar: None. J. Aponte Serrano: None.

\section{P06 Cell Therapy in Solid Tumors}

\section{P06.01 BISPECIFIC ANTIBODY-DRIVEN SYNTHETIC AGONISTIC RECEPTOR - TRANSDUCED T CELLS MEDIATE SPECIFIC AND CONDITIONAL THERAPY IN MELANOMA CANCER MODELS}

${ }^{1} \mathrm{M}$ Benmebarek*, 'J Keyl, ${ }^{1} \mathrm{~F}$ Märkl, ${ }^{2} \mathrm{M}$ Geiger, ${ }^{1} \mathrm{C}$ Karches, 'S Rausch, ${ }^{1} \mathrm{~A}$ Gottschlich, ${ }^{1} \mathrm{~A}$ Öner, ${ }^{1} \mathrm{M}$ Feinendegen, ${ }^{1} \mathrm{~J}$ Dörr, ${ }^{1} \mathrm{~B}$ Cadilha, ${ }^{1} \mathrm{~S}$ Endres, ${ }^{2} \mathrm{C}$ Klein, ${ }^{1} \mathrm{~S}$ Kobold. 'Division of Clinical Pharmacology, Munich, Germany; ${ }^{2}$ Roche Innovation Centre Zurich, Schlieren, Switzerland

\subsection{6/jitc-2020-ITOC7.80}

Background Immunotherapeutic approaches, including immune checkpoint blockade and adoptive T cell therapy (ACT) in the form of tumor-infiltrating lymphocytes (TILs), have had marked success in the treatment of melanoma. Despite these successes, many patients are refractory to treatment or relapse with therapy-resistant disease. To overcome these limitations, we propose a controlled ACT approach, where T cells are armed with synthetic agonistic receptors (SARs) that are conditionally activated only in the presence of a target melanomaassociated antigen, and a cross-linking bispecific antibody (BiAb) specific for both (SAR) T cell and tumour cell.

Materials and Methods A SAR composed of an extracellular EGFRvIII, trans- membrane CD28, and intracellular CD28 and $\mathrm{CD} 3 \mathrm{z}$ domains was fused via overlap- extension PCR cloning. T cells were retrovirally transduced to stably express our SAR construct. We validated our approach in two murine as well as two human cancer models expressing our melanoma-associated target antigens TYRP (murine) and MCSP (human). We confirmed conditional and specific stimulation and proliferation of our $\mathrm{T}$ cells, as well as their tumour-antigen-directed cytotoxicity, in vitro and in vivo.

Results Crosslinking TYRP-EGFRvIII (murine) and MCSPEGFRvIII (human) BiAb, monovalently selective for our SAR, induced conditional antigen-dependent activation, proliferation 